\title{
Greenhouse Gases Observing Satellite (GOSAT) Program Overview and Its Development Status
}

\author{
Masahiro Kasuya $^{1)}$, Masakatsu Nakajima ${ }^{1)}$ and Takashi Hamazaki ${ }^{1)}$ \\ 1) Japan Aerospace Exploration Agency (JAXA), Tsukuba, Japan \\ (Received April 17th, 2008)
}

\begin{abstract}
Greenhouse gases Observing Satellite (GOSAT) is designed and developed to globally monitor the Greenhouse gases distribution from space. This paper presents the program overview and current status of GOSAT.
\end{abstract}

Key Words: GOSAT, Greenhouse Gases, Earth Observing Satellite

\section{Introduction}

Global warming has become a very serious issue for human beings. In February 2005, the 'Kyoto Protocol' came into effect, making it mandatory for developed nations to reduce carbon dioxide emissions by 6 to 8 percent of their total emissions in 1990. The Fourth Assessment Report was issued by the Intergovernmental Panel on Climate Change (IPCC) in 2007, it states that 'Most of the observed increase in global average temperatures since the mid-20th century is very likely due to the observed increase in anthropogenic Green House Gases (GHG) concentrations, ${ }^{\text {, }}$.

Greenhouse gases Observing Satellite (GOSAT) scheduled to be launched in Fiscal Year 2008 is designed and developed to globally monitor the GHGs distribution, particularly Carbon Dioxide $\left(\mathrm{CO}_{2}\right)$ and the Methane $\left(\mathrm{CH}_{4}\right)$, from space.

GOSAT is a cooperative project between Japan Aerospace Exploration Agency (JAXA), the Ministry of Environment (MOE), and the National Institute for Environmental Studies (NIES).

This paper presents the program overview and current status of GOSAT.

\section{Program overview}

\subsection{Background}

It is becoming apparent that the increase in green house gases due to human activities causes global warming which leads to climate change, and rise of average temperature as well as that of sea levels.

In order to understanding the global warming mechanisms and to promote its countermeasures, it is necessary to grasp the concentration and increase/decrease in green house gases at various locations around the world.

As shown in the Fig.1, currently there are 256 Green House Gases observing stations (as of Apr. 2008) on the ground in operation worldwide, and the collected data from these stations are managed by the World Data Centre for Greenhouse Gases (WDCGG).

However, the number of ground stations is quite limited, and they are scattered unevenly around the world.

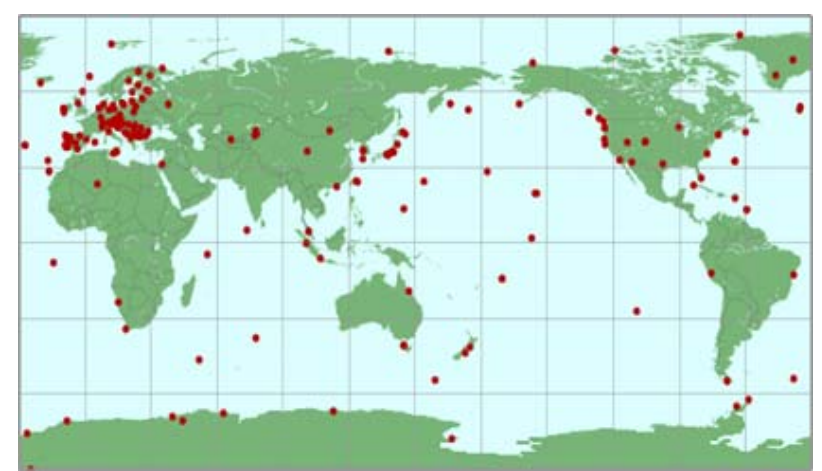

Fig.1 Diagram of global observation points of GHGs ${ }^{2)}$

(By WMO-WDCGG)

On the other hand, the Fig.2 shows observing points in the case of GOSAT in orbit. GOSAT is designed to provide 56,000 observation points and obtain frequent data from the same region every 3 days with a unique sensor.

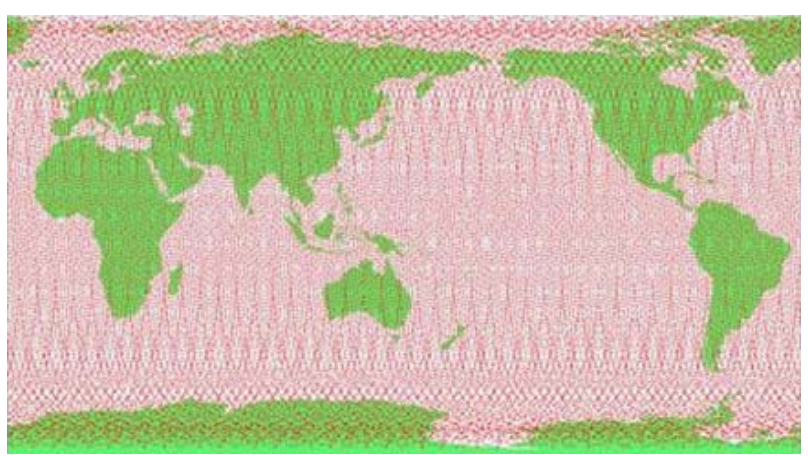

Fig.2 Observation points from GOSAT

\subsection{Mission Objectives}

One of the main objectives of the GOSAT mission is to contribute to environmental administration by estimating the 
GHGs net emissions at 'Sub-continental' scale.

The other objective is to make contribution in the advancement of earth observation technologies for future missions such as high reliability bus-system.

\subsection{Mission Targets}

In order to achieve above objectives, several mission targets have been defined as followings.

One of the mission targets is to make observation of $\mathrm{CO}_{2}$ density in 3 months average with $1 \%$ (4ppmv) relative accuracy in $1000 \mathrm{~km}$ spatial resolution during the first commitment period (2008 to 2012) of the Kyoto Protocol.

Other mission target is the reduction of estimation errors by half in identifying the GHGs net emissions at Sub-continental scale with the data obtained by GOSAT in conjunction with the data gathered by the ground instruments.

\subsection{Organizations}

GOSAT is a joint project by JAXA, MOE and NIES as shown in the Fig.3. JAXA is responsible for satellite development, launch, satellite operation, data acquisition operation and calibration, and JAXA and MOE are in charge of the sensor development, while MOE and NIES are responsible for satellite data utilization.

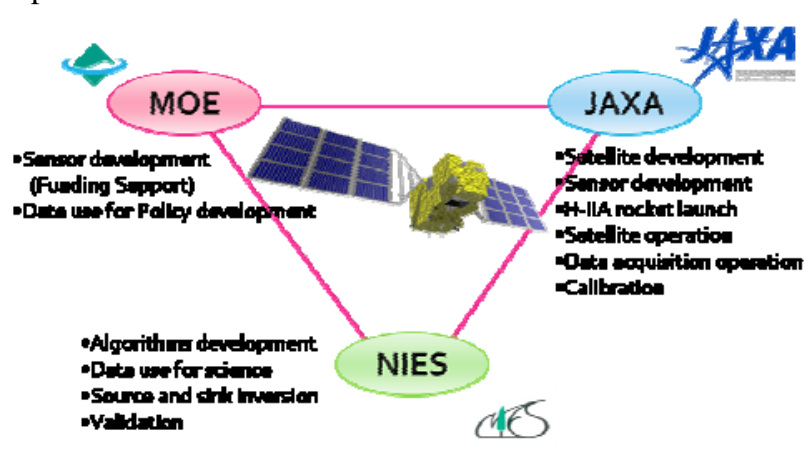

Fig.3 Organizations of GOSAT project

\subsection{Schedule}

GOSAT is scheduled to be launched by Japanese H-IIA launch vehicle in Fiscal Year 2008.

The initial check-out activities of satellite instruments will be completed by approximately 3 months after launch, and initial in-flight calibration will be carried out between 3 and 6 months after launch.

\section{Spacecraft}

\subsection{Main characteristics of GOSAT}

GOSAT is a medium-size satellite which weighs $1750 \mathrm{~kg}$ with the size of $3.7 \mathrm{~m}$ in height, $1.8 \mathrm{~m}$ in width, and $2.0 \mathrm{~m}$ in depth. GOSAT has two solar array paddles with the wing span of $13.7 \mathrm{~m}$. The Fig.4 illustrates the image of GOSAT in orbit, and as shown in the Table 1 the satellite is placed in the sun-synchronous orbit of $666 \mathrm{~km}$ altitude at 13:00 local time with an inclination angle of 98 degree.

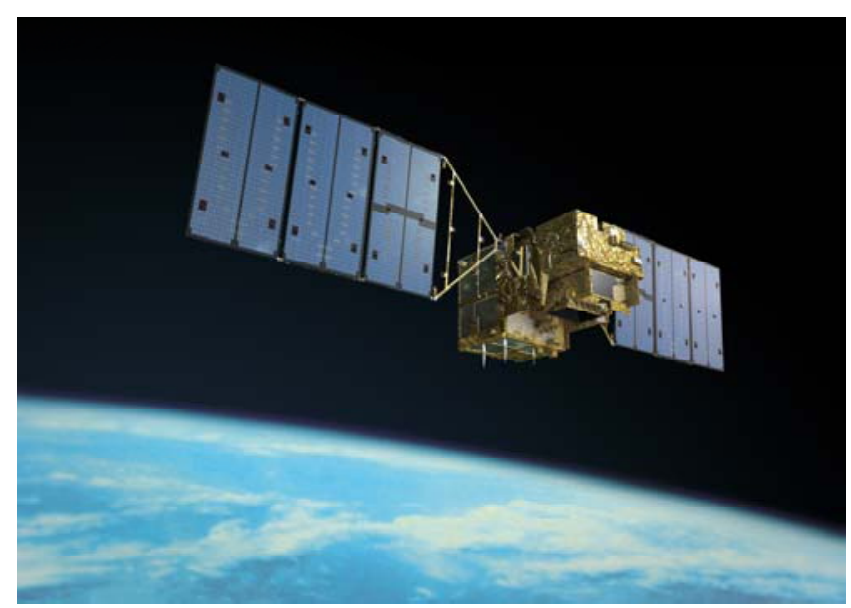

Fig.4 Illustration of GOSAT in Orbit

Table 1 Specification summary of GOSAT

\begin{tabular}{|l|l|l|}
\hline \multirow{2}{*}{ Size } & Main body & $3.7 \mathrm{~m}(\mathrm{H}) \times 1.8 \mathrm{~m}(\mathrm{~W}) \times 2.0 \mathrm{~m}(\mathrm{D})$ \\
\cline { 2 - 3 } & Wing span & $13.7 \mathrm{~m}$ \\
\hline Mass & Total & $1,750 \mathrm{~kg}$ \\
\hline Power & Total & $3.8 \mathrm{~kW}$ \\
\hline Life Span & & 5 years \\
\hline \multirow{4}{*}{ Orbit } & & Sun Synchronous Orbit \\
\cline { 2 - 3 } & Local time & $13: 00 \pm 0: 15$ \\
\cline { 2 - 3 } & Attitude & $666 \mathrm{~km}$ \\
\cline { 2 - 3 } & Inclination & $98 \mathrm{deg}$ \\
\cline { 2 - 3 } & Re-visit & 3 days \\
\hline \multirow{3}{*}{ Launch } & Vehicle & H-IIA \\
\cline { 2 - 3 } & Schedule & JFY 2008 \\
\hline \multirow{2}{*}{$\begin{array}{l}\text { Downlink station } \\
\text { (mission data) }\end{array}$} & $\begin{array}{l}\text { Hatoyama(Japan) } \\
\text { Svalbard(Norway) }\end{array}$ \\
\hline
\end{tabular}

\subsection{Mission instruments}

Two mission instruments are carried onboard by GOSAT as shown in the Fig. 5.

The main instrument; Thermal And Near infrared Sensor for carbon Observation Fourier Transform Spectrometer (TANSO-FTS), is a Fourier Transform Spectrometer with high optical throughput, high spectral resolution, and wide spectral coverage as shown Fig.6. TANSO-FTS is capable of detecting the spectra of solar Short Wave Infrared Spectra (SWIR) reflected and scattered on the earth's surface as well as the Thermal Infra-Red spectra (TIR) radiated from the ground and the atmosphere. These spectra include the absorption lines of GHG such as carbon dioxide $\left(\mathrm{CO}_{2}\right)$ and methane $\left(\mathrm{CH}_{4}\right)$. The Fig. 6 shows spectral coverage of TANSO-FTS.

By SWIR observation, it is able to monitor the column density of GHG at daytime and over land-regions. While, by TIR observation, it is able to monitor the column density of GHG at nighttime and over sea-regions as well as to estimate the attitude distribution of GHG. 
As for an auxiliary sensor, TANSO Cloud and Aerosol Imager (TANSO-CAI) is attached aside the TANSO-FTS. TANSO-CAI is a radiometer of ultraviolet (UV), visible, and SWIR which purpose is to correct and compensate for cloud and aerosol interference.

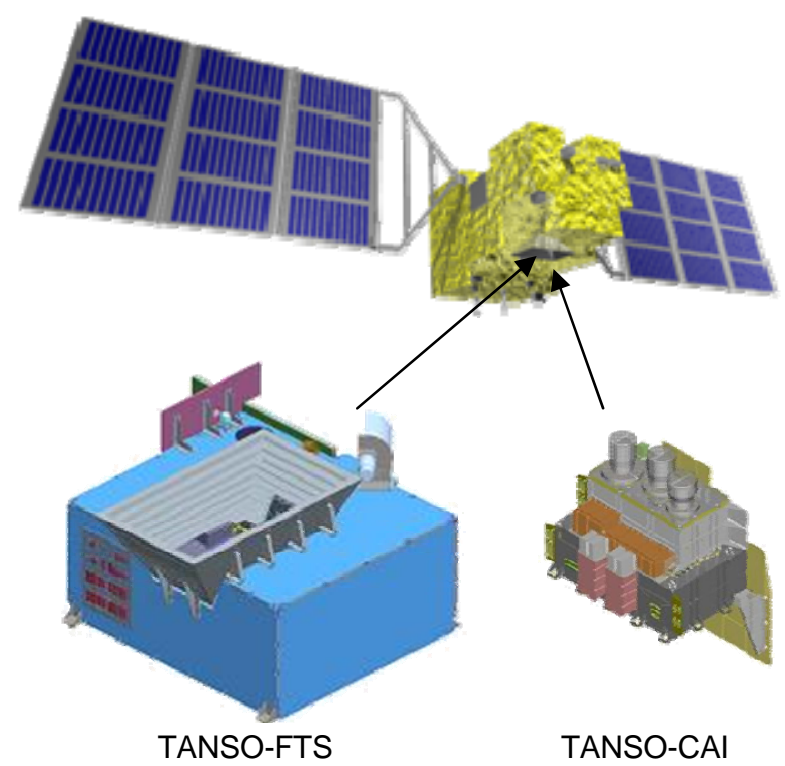

Fig.5 Mission instruments of GOSAT

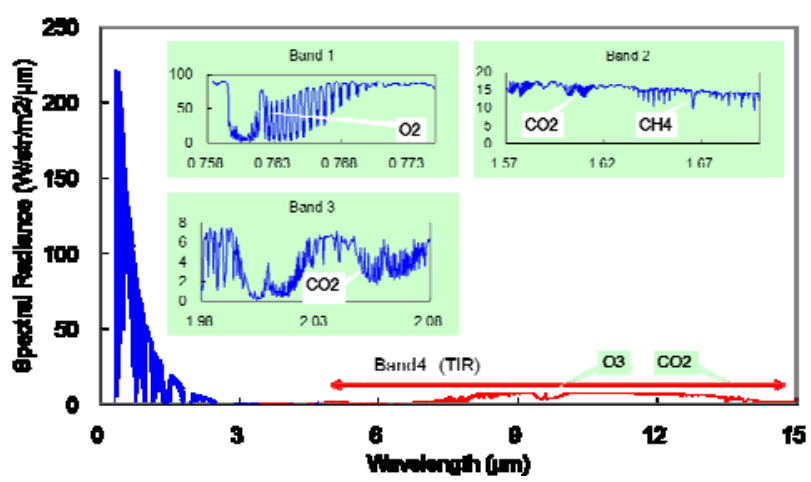

Fig.6 Spectral coverage of TANSO-FTS

\subsection{Monitor Camera (CAMs)}

Eight (8) Monitor-cameras (CAMs) are accommodated on GOSAT. The Fig.7 and Fig. 8 show the flight model of CAM and their location on GOSAT. CAM is designed to monitor the spacecraft exterior in orbit. CAMs are capable of capturing clear images during the eclipse using LED lights.

The images acquired by CAMs are used to grasp the satellite status accurately (e.g. deployment status of the solar array paddles, contamination during the rocket fairing separation event), and also play important roles by responding to anomalies promptly. The Fig.9 illustrates 'expected images' taken by CAMs (drawn by CAD software).

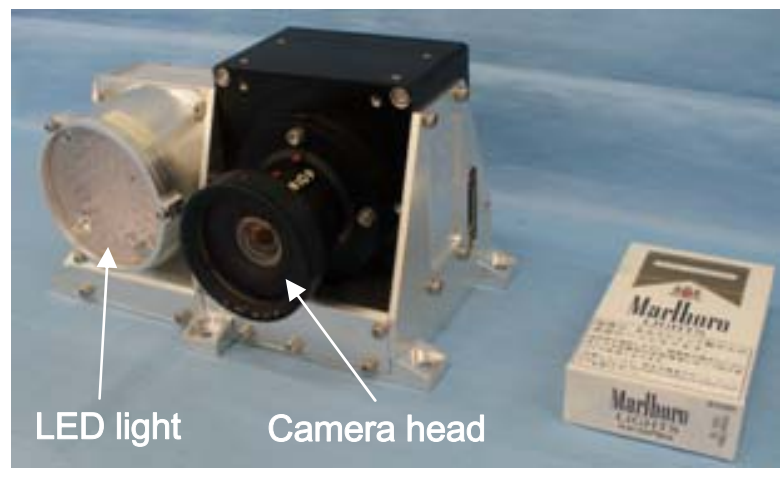

Fig.7 Monitor camera (CAM)
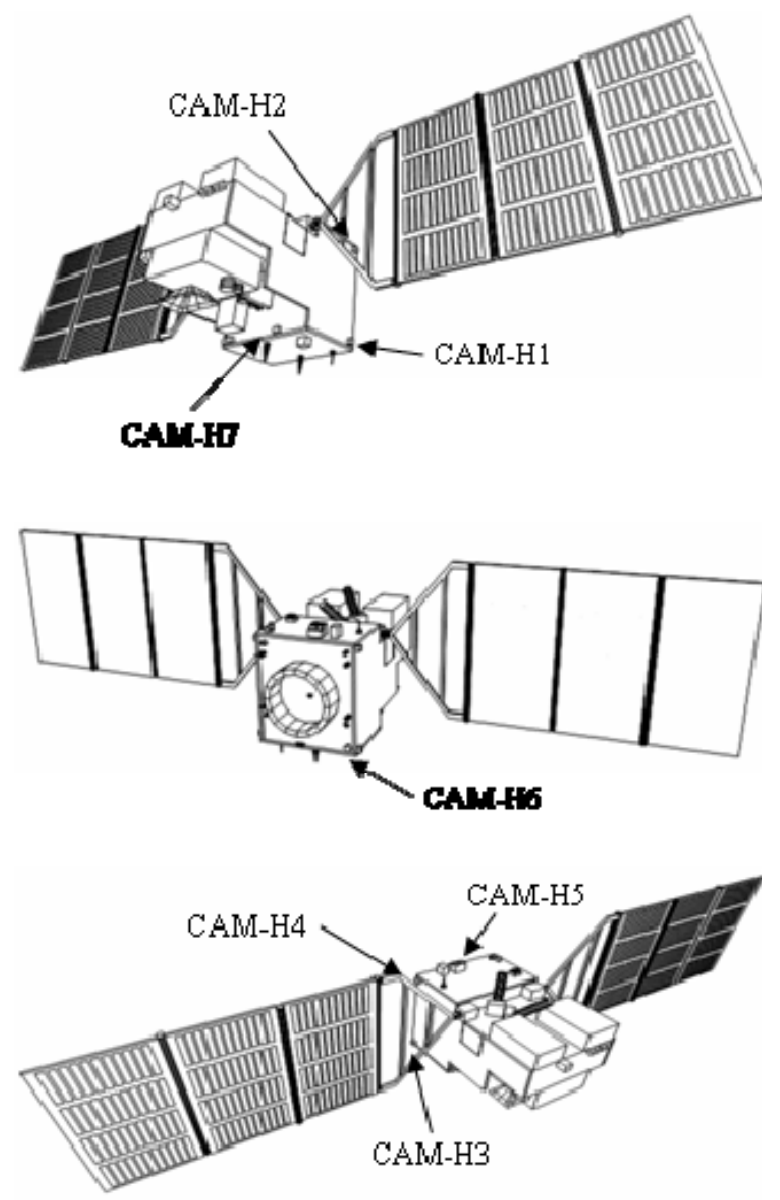

Fig.8 Attached locations of CAMs 


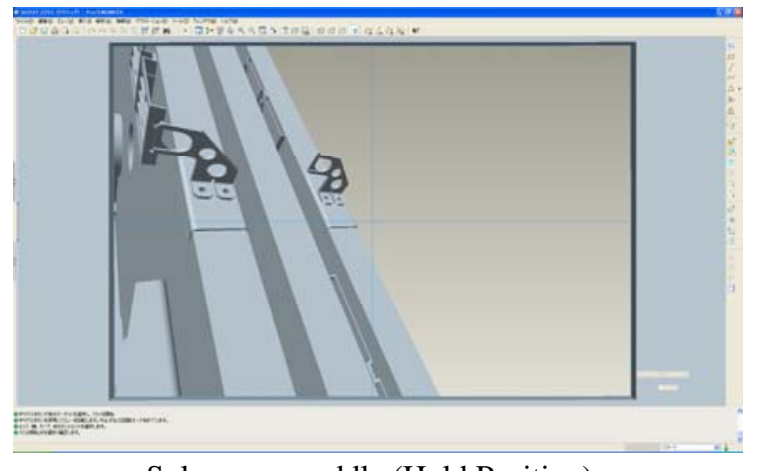

Solar array paddle (Hold Position)

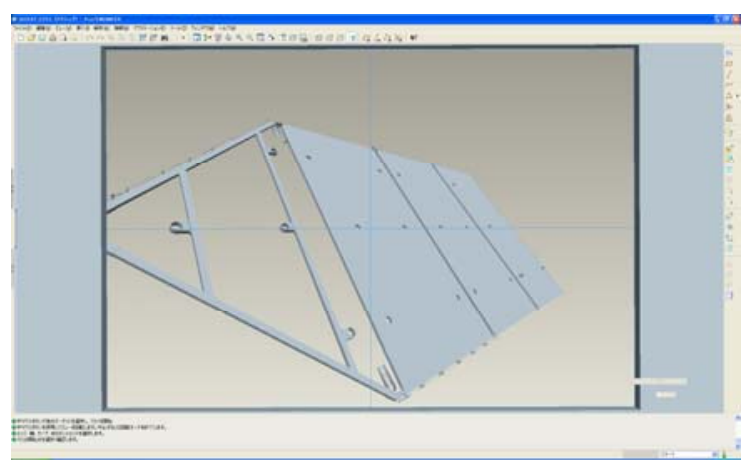

Solar array paddle (Deployed Position)

Fig.9 Expected images by CAMs (drawn by CAD)

\subsection{Technical Data Acquisition Equipment (TEDA)}

JAXA has long establishment in developing on-board space environment measurement systems named Technical Data Acquisition Equipment (TEDA), and continued monitoring of the orbital radiation environment.

As shown in the Fig.10 and Fig.11, TEDA consists of four Light particle telescope (LPT1-4) and one Heavy Ion Telescope (HIT). LPT discriminates electrons, protons, and alpha particles and analyzes their quantitative energy, while HIT characterizes the fluxes and energy distributions of heavy ions having masses from that of helium (He) to iron (Fe).
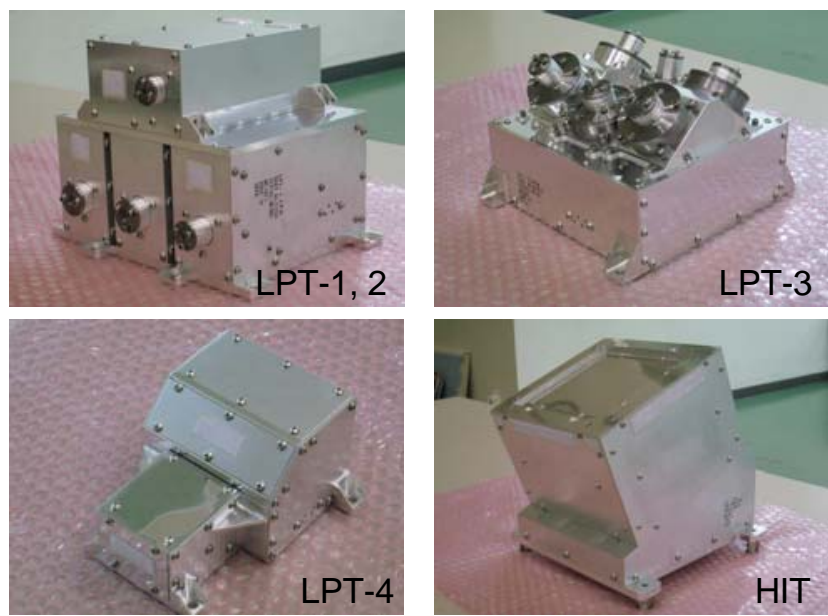

Fig.10 Flight models of GOSAT’s TEDA

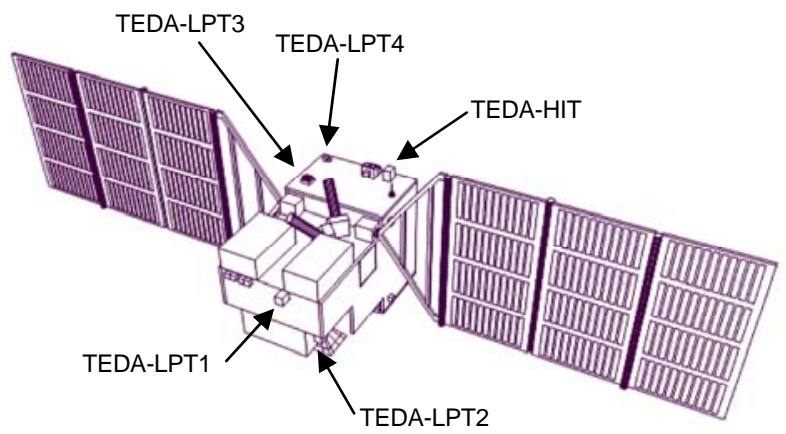

Fig.11 Attached locations of TEDA

\section{Designing and development policy}

JAXA experienced unfortunate failures of the solar array paddle and power system on the Earth observing satellites ADEOS and ADEOS-II in 1997 and 2003. GOSAT happen to be the 'first satellite' that JAXA initiated its development following these setbacks. Under the circumstance, 'Achieving High Reliability' is designated as the 'first priority' for the 'designing and development policy' of GOSAT.

In order to achieve highly reliable satellite, GOSAT adopted further enhancement in robustness and survivability with the following techniques and countermeasures:

- Maximum utilization of flight-proven components

- Complete eliminate of single point failure

- Strategic adoption of functional redundancy

- Thorough mission-duty-cycle tests

\subsection{Maximum utilization of flight-proven components}

GOSAT bus-system consists of about 50 types of components. In order to sustain the guaranteed reliability, GOSAT selected most of the components from the earlier satellites with flight-proven records.

\subsection{Complete elimination of single point failure}

The GOSAT project provided redundancy to the components including ones that conventionally considered highly reliable in order to maintain the minimum mission operation in the failure event on the primary component, such as dual solar array paddles, dual power system lines, dual X-band antenna and dual S-band antennas.

For instance, as shown in Fig.12, a single X-band antenna and an X-band switch/filter are equipped for many of the past satellite configuration which are distinguished as components with single failure points; however, that may also lead to a total mission lost. GOSAT is designed to provide all the components redundant for the $\mathrm{X}$-band transmission subsystem including $\mathrm{X}$-band antenna as shown in Fig.13 so as to continue the mission data transmission in the failure event on the primary component. 


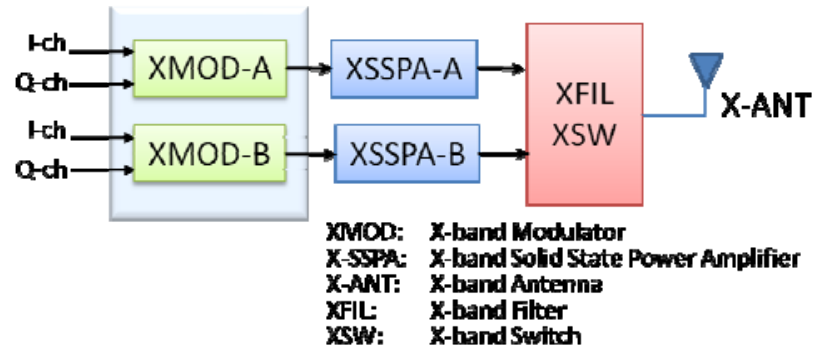

Fig.12 X-band transmission subsystem (Past satellite)

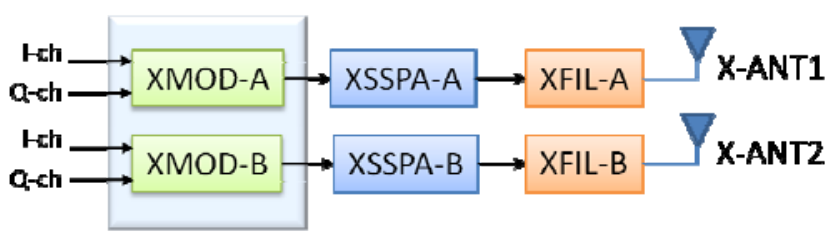

Fig.13 X-band transmission subsystem (GOSAT)

\subsection{Strategic adoption of functional redundancy}

The GOSAT project adopted functional redundancy for mission-critical subsystem to maintain the minimum mission operation during the simultaneous failure on both the primary and redundant components due to designing or manufacturing anomaly.

For example, GOSAT is capable of transmitting House -Keeping telemetry to the ground stations from both S-band and X-band data transmission systems.

\subsection{Mission duty cycle tests for two times duration}

The critical mechanical components were subjected to mission duty cycle tests for two times the mission duration to ensure the margin of lifetime; five-years of mission operation.

\section{Current development status}

System Proto-flight model (PFM) tests for GOSAT are underway at Tsukuba Space Center, JAXA. Fig.14 displays the current configuration of GOSAT system proto-flight model during testing.

The spacecraft integration and initial electric performance tests which started from October 2007 were completed at the end of March 2008. GOSAT is currently prepared for the thermal and vacuum test and scheduled to be carried out from the middle of May to the end of June 2008. Following the thermal and vacuum test, acoustic test, vibration test, and final electric performance test are scheduled to be completed by the end of October 2008.

After completion of all system proto-flight model tests, GOSAT will be shipped to Tanegashima Space Center (Fig.15), the launch site of Japanese H-IIA rocket (Fig.16), and prepared for the launch within Japanese fiscal year 2008.

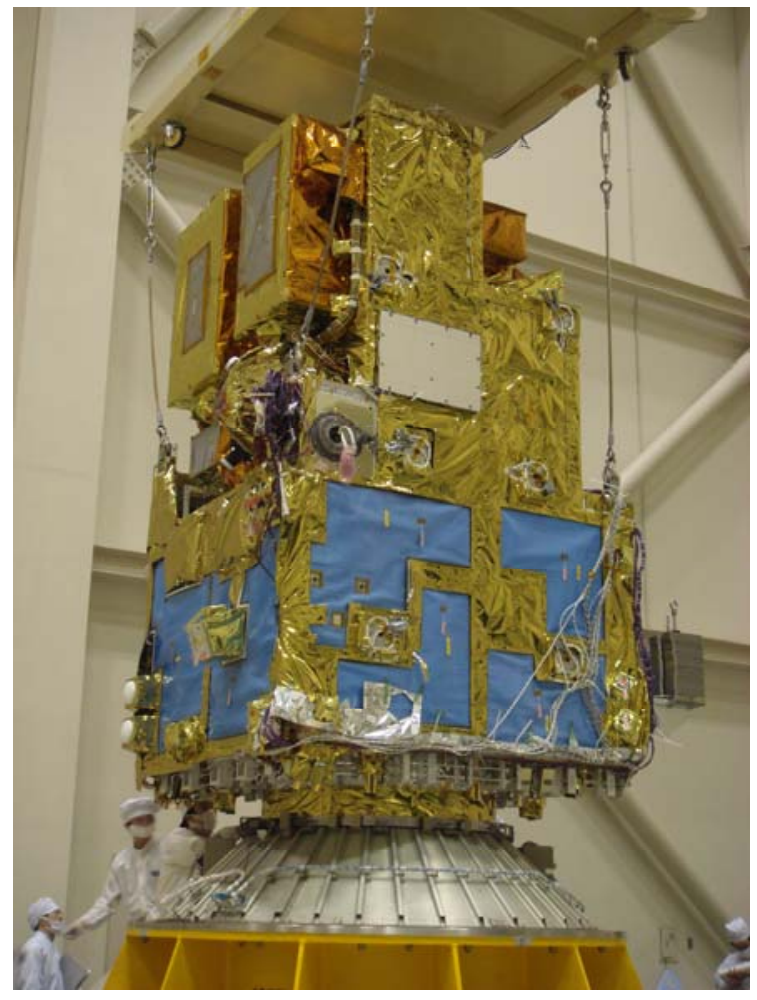

Fig.14 GOSAT system proto-flight model (PFM)

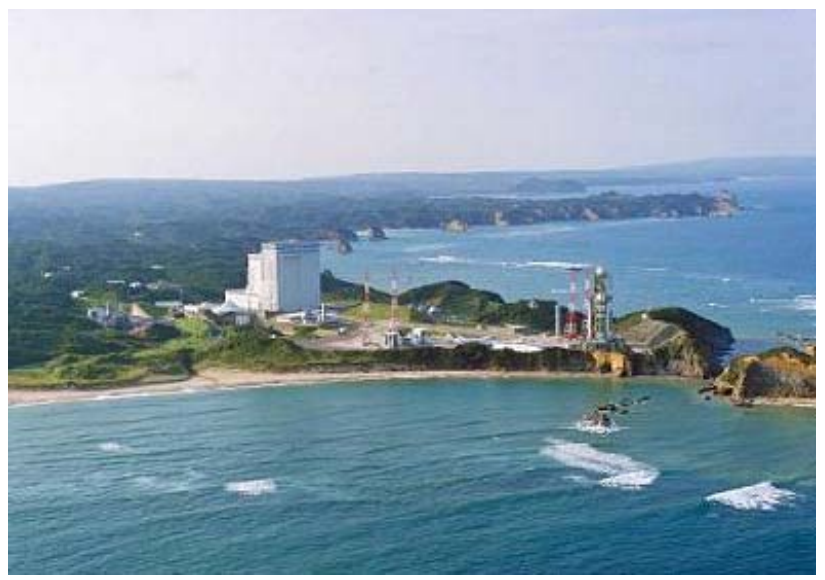

Fig,15 Launch site in Tanegashima Space Center

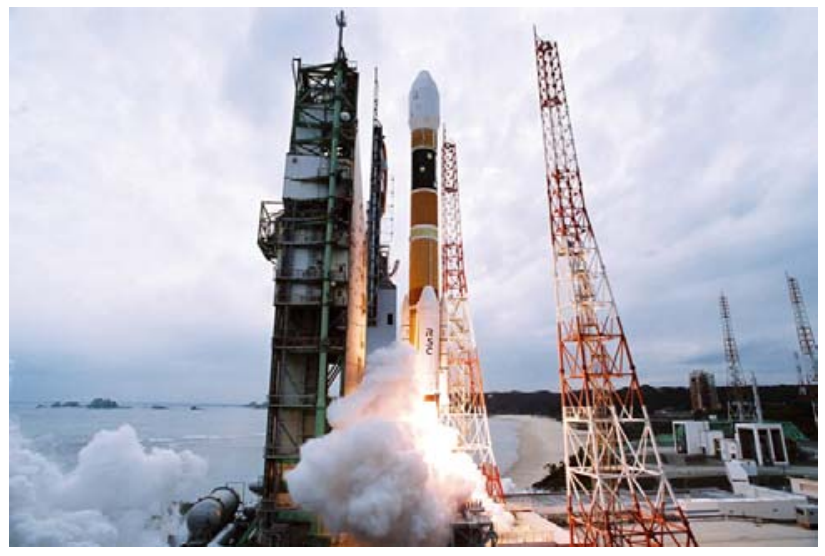

Fig.16 H-IIA launch vehicle 


\section{Conclusion}

This paper presented the overview and current status of GOSAT from the satellite specifics to the design \& development strategies. With the GOSAT schedule steadily advanced to the final development phase, JAXA and the GOSAT project team are getting ready and bracing up for the GOSAT mission success.

\section{References}

1) IPCC, 2007: Climate Change 2007: Synthesis Report. Contribution of Working Groups I, II and III to the Fourth Assessment Report of the Intergovernmental Panel on Climate Change [Core Writing Team, Pachauri, R.K and Reisinger, A.(eds.)]. IPCC, Geneva, Switzerland,

2) http://gaw.kishou.go.jp/wdcgg/jp/wdcgg_j.html 